\title{
Facilidades e dificuldades na implantação do Programa Saúde na Escola em um município do nordeste do Brasil
}

\section{Facilities and difficulties in implementing the Health at School Program in a municipality in northeastern Brazil}

\author{
Facilidades y dificultades en la implantación del Programa Salud en la \\ Escuela en un municipio del nordeste de Brasil
}

\section{Eliabe Rodrigues de Medeiros ${ }^{1}\left({ }^{-}\right.$, Erika Simone Galvão Pinto ${ }^{2}\left({ }^{-}\right.$, Alany Carla de Sousa Paiva ${ }^{3}(\mathbb{D}$, Camila Priscila Abdias do Nascimento ${ }^{4}{ }^{\circledR}$, Danielle Gonçalves da Cruz Rebouças ${ }^{5}{ }^{(}$, Sandy Yasmine Bezerra e Silva ${ }^{6}$ (D)}

\section{Histórico}

Recibido:

15 de febrero de 2018

Aceptado:

10 de abril de 2018

1 Doutorando em Enfermagem, Universidade Federal do Rio Grande do Norte. Natal, RN, Brasil. Autor de Correspondência. E-mail: eliabe.medeiros@hotmail.com

2 Doutora em Enfermagem, Professora Adjunta do Departamento de Enfermagem da Universidade Federal do Rio Grande do Norte. Natal, RN, Brasil. 3 Especializanda em Docência no Ensino Superior Universidade Potiguar. Natal, RN, Brasil.

4 Especializanda em Saúde Pública, Universidade Potiguar. Natal, RN, Brasil.

5 Especializanda em Saúde Pública: Enfermagem Oncológica, Escola da Assembleia Legislativa do Rio Grande do Norte. Natal, RN, Brasil.

6 Mestranda em Enfermagem, Universidade Federal do Rio Grande do Norte. Natal, RN, Brasil.
Introducão: A saúde escolar no Brasil é ofertada através do Programa Saúde na Escola. Neste estudo objetivou-se identificar as facilidades e dificuldades na implantação do Programa Saúde na Escola em município do nordeste brasileiro. Materiais e Métodos: Pesquisa descritiva, transversal, quantitativa, realizada com 105 profissionais da Estratégia Saúde da Família que já tinham desenvolvido atividades do programa. A coleta de dados foi realizada por meio de instrumento com perguntas abertas e fechadas. Utilizou-se as questões abertas referentes às facilidades e dificuldades enfrentadas na implantação do Programa Saúde na Escola. Os dados foram analisados por meio da estatística descritiva e apresentados em números absolutos e relativos. Resultados: As facilidades mais frequentes são a articulação intersetorial $(38,1 \%)$ e a satisfação profissional $(24,8 \%)$ em executar as atividades do Programa Saúde na Escola. Já as dificuldades foram: escassez de recursos materiais e financeiros $(50,5 \%)$, ausência de articulação intersetorial $(26,7 \%)$ e excesso de atividades no processo de trabalho $(17,1 \%)$. Discussão: Foi observada maior frequência de dificuldades em relação às facilidades e os resultados encontrados assemelham-se e distanciam-se de outros estudos da área. Conclusões: A satisfação dos profissionais e a articulação entre os setores saúde e educação são elementos facilitadores na implantação do Programa Saúde na Escola. No entanto, insuficiência de recursos materiais e financeiros e excesso de atribuições no processo de trabalho são exemplos de fatores que comprometem sua implantação.

Palavras chave: Saúde; Educação; Serviços de Saúde Escolar; Atenção Primária à Saúde; Promoção da Saúde.

Introduction: School health in Brazil is offered through the School Health Program. The purpose of this study was to identify the facilities and difficulties in implementing the Health at School Program in a municipality in the Northeast of Brazil. Materials and Methods: Descriptive, cross-sectional quantitative research conducted with 105 professionals from the Family Health Strategy who had already carried out activities of the program. Data was collected through an instrument with open and closed questions. We used open questions regarding the facilities and difficulties encountered in implementation of the Health Program in the School. Data were analyzed through descriptive statistics and presented in absolute and relative numbers. Results: The most frequent facilities are intersectoral articulation $(38.1 \%)$ and professional satisfaction $(24.8 \%)$ in performing the activities of the Health Program in the School. The difficulties were: shortage of material and financial resources $(50.5 \%)$, lack of intersectoral articulation $(26.7 \%)$, and excess of activities in the work process $(17.1 \%)$. Discussion: A greater frequency of difficulties was observed in relation to the facilities and the results found show similarity and distance themselves from other studies of the area. Conclusions: The satisfaction of professionals and articulation between the health and education sectors are facilitating elements in implementing the Health at School Program. However, insufficient material and financial resources, as well as excess attributions in the work process are examples of factors that jeopardize its implementation.

Key words: Health; Education; School Health Services; Primary Health Care; Health promotion.

Resumen

Introducción: La salud escolar en Brasil es ofrecida a través del Programa Salud en la Escuela. El objetivo de este estudio fue identificar las facilidades y dificultades en la implantación del Programa Salud en la Escuela en un municipio del nordeste brasilero. Materiales y Métodos: Investigación descriptiva, transversal, cuantitativa, realizada con 105 profesionales de la Estrategia Salud de la Familia que ya habían desarrollado actividades del programa. La recolección de datos fue realizada por medio de un instrumento con preguntas abiertas y cerradas. Se utilizaron las preguntas abiertas referentes a las facilidades y dificultades enfrentadas en la implantación del Programa Salud en la Escuela. Los datos fueron analizados por medio de la estadística descriptiva y presentados en números absolutos y relativos. Resultados: Las facilidades más frecuentes son la articulación intersectorial $(38.1 \%$ ) y la satisfacción profesional $(24.8 \%)$ en ejecutar las actividades del Programa Salud en la Escuela. Las dificultades fueron: escasez de recursos materiales y financieros (50.5\%), ausencia de articulación intersectorial $(26.7 \%)$ y exceso de actividades en el proceso de trabajo (17.1\%). Discusión: Se observó mayor frecuencia de dificultades en relación a las facilidades y los resultados encontrados se asemejan y se distancian de otros estudios del área. Conclusiones: La satisfacción de los profesionales y la articulación entre los sectores salud y educación son elementos facilitadores en la implantación del Programa Salud en la Escuela. Sin embargo, la insuficiencia de recursos materiales y financieros y exceso de atribuciones en el proceso de trabajo son ejemplos de factores que comprometen su implementación.

Palabras clave: Salud; Educación; Servicios de Salud Escolar; Atención Primaria a la Salud; Promoción de la Salud.

Como citar este artigo: Medeiros ER, Pinto ESG, Paiva ACS, Nascimento CPA, Rebouças DGC, Silva SYB. Facilidades e dificuldades na implantação do Programa Saúde na Escola em um município do nordeste do Brasil. Rev Cuid. 2018; 9(2): 2127-34. http://dx.doi.org/10.15649/cuidarte.v9i2.514

(c) (i) (C)2018 Universidad de Santander. Este es un artículo de acceso abierto, distribuido bajo los términos de la licencia Creative Commons Attribution (CC BY-NC 4.0), que permite el uso ilimitado, distribución y reproducción en cualquier medio, siempre que el autor original y la fuente sean debidamente citados. 


\section{INTRODUÇÃO}

A promoção da saúde escolar propõe que atividades focalizadas na melhoria da saúde e bem estar da comunidade escolar (educandos, professores e outros funcionários da escola) sejam realizadas. As atividades devem ser realizadas em parcerias com a comunidade e escolas para incentivar o empoderamento dos sujeitos ${ }^{1}$.

No Brasil, a saúde escolar é ofertada através do Programa Saúde na Escola, principal ação programática direcionada à estes espaços que tem como finalidade a atenção integral a saúde dos estudantes através de ações de prevenção de doenças e agravos, promoção e atenção à saúde deste grupo. As ações devem acontecer nas escolas e Unidades de Saúde da Família (USF) dos seus territórios por meio da criação de vínculo dos profissionais de saúde com os membros da comunidade escolar ${ }^{2}$.

A importância desta intervenção é ressaltada ao se observar que, no ano de $2015,55,5 \%$ dos estudantes brasileiros entre 13 e 17 anos de idade já haviam experimentado álcool, outros $18,4 \%$ disseram ter utilizado tabaco, 27,5\% iniciaram atividade sexual e 19,8\% afirmaram ter praticado bullying entre os colegas ${ }^{3}$. Estas e outras situações de risco à saúde dos estudantes podem ser prevenidas a partir das atividades do Programa Saúde na Escola, motivo pelo qual reforça a necessidade nos diversos municípios brasileiros valorizar as ações de promoção a saúde.

O que observa-se em todas as macrorregiões brasileiras são equipes de Atenção Primária à Saúde executando atividades que são propostas pelo Programa Saúde na Escola com destaque para as ações que buscam a promoção da saúde além da prevenção de doenças e agravos ${ }^{4}$.

No entanto, a implantação de um programa de abrangência nacional e de caráter intersetorial pode ter influência de diversificados fatores, como é o caso da diversidade de situações políticas e organizacionais, que podem comprometer seu processo de implantação, compreendido pela adequada operacionalização de determinada intervenção ${ }^{5}$.

Deste modo, questiona-se quais fatores dificultam ou facilitam este processo de implantação. São escassos os estudos que se detêm a identificar tais informações, motivo pelo qual, conhecêlos possibilitará que a gestão e profissionais do Programa Saúde na Escola reforcem os aspectos positivos e readéquem as ações propostas a fim de corrigir as situações que comprometem a implantação do programa.

Frente ao exposto, objetiva-se identificar as facilidades e dificuldades na implantação do Programa Saúde na Escola em município do nordeste brasileiro.

\section{MATERIAIS E MÉTODOS}

Pesquisa descritiva, transversal, de abordagem quantitativa, que seguiu os itens da Declaração $\mathrm{STROBE}^{6}$, em sua construção. O estudo foi realizado no Município de Natal, capital do Rio Grande do Norte, estado do nordeste brasileiro, município este que tem seus serviços de saúde organizados em cinco distritos sanitários (Sul, Leste, Oeste, Norte I e Norte II). O atendimento à população pela Estratégia Saúde da Família 
é realizado em 38 USF. As atividades do Programa Saúde na Escola são executadas pelos profissionais de saúde que atuam nestas unidades, quais sejam: médicos, enfermeiros, técnicos ou auxiliares em enfermagem, agente comunitário de saúde, odontólogo e auxiliar ou técnico em saúde bucal.

Para definição da população considerou-se que cada USF tem no mínimo um profissional de cada categoria, perfazendo um total de 228 profissionais de saúde. A definição da amostra foi realizada com base no cálculo amostral para populações finitas ao considerar o erro amostral de $5 \%$ e intervalo de confiança de $95 \%$. Obteve-se que participariam 144 profissionais, seis por USF (um de cada categoria profissional), perfazendo um total de 24 USF, desde que respeitasse o critério de inclusão de já ter participado das atividades de saúde na escola.

A coleta de dados aconteceu entre os meses de maio e julho de 2017 e foi realizada por acadêmicos de enfermagem e enfermeiros através de questionário estruturado de indagações referentes à caracterização dos profissionais e outras sobre as dificuldades e facilidades que interferem na implantação do Programa Saúde na Escola. O ambiente onde aconteceu a coleta foi na USF.

Os dados foram organizados e agrupados respeitando o critério de semelhança. Isto foi realizado com auxílio do IBM SPSS Statistics e os resultados apresentados em tabelas por meio da estatística descritiva em números absolutos e relativos.
Por tratar-se de um pesquisa envolvendo seres humanos, respeitou-se as preconizações éticas ressaltadas na resolução do Conselho Nacional de Saúde de número 466/2012, onde foi necessária a apreciação por parte do Comitê de Ética em Pesquisa de uma instituição de ensino superior pública, a Universidade Federal do Rio Grande do Norte. O colegiado aprovou sua execução mediante o parecer de número 2.064.901 e Certificado de Apresentação para Apreciação Ética 64873916.1.0000.5537. Aos participantes foi explicado o estudo, apresentados os riscos e requerido que o Termo de Consentimento Livre e Esclarecido fosse assinado.

\section{RESULTADOS}

Participaram do estudo 105 profissionais de saúde, onde $6(5,7 \%)$ eram médicos, $20(19,0 \%)$ enfermeiros, $13(12,4 \%)$ auxiliares ou técnicos em enfermagem, 24 (22,9\%) agentes comunitários de saúde, 22 (21,0\%) odontólogos e 20 (19,0\%) auxiliares ou técnicos em saúde bucal. Maior parte deles eram do sexo feminino $(84,8 \%)$.

Referente à qualificação profissional, 17 (16,2\%) haviam concluído o ensino médio, 33 (31,4\%) possuíam formação técnica, 20 (19,0\%) eram graduados, $28(26,7 \%)$ possuíam formação a nível de especialização, $5(4,8 \%)$ mestrado e 2 $(1,9 \%)$ doutorado. Estes profissionais exerciam suas profissões há uma média de 22,3 anos. No entanto, atuavam na ESF em média, 12,1 anos. A participação nas ações de saúde na escola era exercida há, em média, 7,2 anos.

Os fatores facilitadores referidos pelos profissionais estão apresentados na Tabela 1 . 
Tabela 1. Facilidades na implantação do Programa Saúde na Escola. Natal, RN, Brasil, 2017

\begin{tabular}{|l|c|c|}
\hline Facilidades & $\mathbf{N}$ & $\mathbf{\%}$ \\
\hline Articulação intersetorial & 40 & 38,1 \\
\hline Satisfação profissional & 26 & 24,8 \\
\hline Continuidade do cuidado & 8 & 7,6 \\
\hline Disponibilidade dos recursos materiais e financeiros & 7 & 6,7 \\
\hline Atuação multiprofissional & 7 & 6,7 \\
\hline Aceitabilidade das atividades & 2 & 1,9 \\
\hline Proximidade territorial da escola & 1 & 1,0 \\
\hline
\end{tabular}

Fonte: dados da pesquisa, 2017.

O fator mais frequente apontado pelos participantes foi a articulação intersetorial $(38,1 \%)$ o que ressalta a integração entre os profissionais dos setores saúde e de educação, como é o caso dos professores, coordenadores pedagógicos e gestores das escolas. Com frequência inferior, porém representativa, foi apontada à satisfação profissional (24,8\%) em executar as atividades do Programa Saúde na Escola além da possibilidade de oferecer continuidade ao cuidado $(7,6 \%)$ para com educandos que necessitam de atenção além do ambiente escolar.

As dificuldades estão apresentadas na Tabela 2.

Tabela 2. Dificuldades na implantação do Programa Saúde na Escola. Natal, RN, Brasil, 2017

\begin{tabular}{|l|c|c|}
\hline Dificuldades & N & \% \\
\hline Escassez de recursos materiais e financeiros & 53 & 50,5 \\
\hline Desarticulação intersetorial & 28 & 26,7 \\
\hline Excesso de atividades no processo de trabalho & 18 & 17,1 \\
\hline Carência de incentivo da gestão & 11 & 10,5 \\
\hline Descontinuidade do cuidado & 11 & 10,5 \\
\hline Locomoção à escola & 8 & 7,6 \\
\hline Infraestrutura inadequada & 7 & 6,7 \\
\hline Desinteresse dos estudantes & 7 & 6,7 \\
\hline Inexistência de capacitações & 7 & 6,7 \\
\hline Desinteresse dos profissionais de saúde & 6 & 5,7 \\
\hline Ausência de escola no território & 4 & 3,8 \\
\hline Falta de contribuição dos pais & 3 & 2,9 \\
\hline Recursos materiais inadequados & 2 & 1,9 \\
\hline Ausência de atuação multiprofissional & 2 & 1,9 \\
\hline Resistências éticas e legais & 2 & 1,9 \\
\hline
\end{tabular}

Fonte: dados da pesquisa, 2017. 
Observou-se que a dificuldade mais relatada foi a escassez de recursos materiais e financeiros $(50,5 \%)$ para subsidiar a execução das atividades do programa os quais são obtidos, na maioria das vezes, pelos próprios profissionais. Também foi referida como dificuldade a desarticulação intersetorial $(26,7 \%)$ de modo que as ações são prevalentemente realizadas pelos profissionais de saúde. Estes profissionais têm o excesso de atividades no processo de trabalho $(17,1 \%)$ nas USF como dificuldade que impacta negativamente na execução das ações de saúde escolar.

\section{DISCUSSÃO}

Os fatores que facilitaram a implantação do Programa Saúde na Escola referem-se à execução de atividades com a participação articulada entre os profissionais dos setores saúde e educação, a satisfação em executar estas atividades, assim como a possibilidade de oferecer a continuidade do cuidado aos estudantes. A contribuição e participação multiprofissional e a disponibilidade de recursos materiais e financeiros também incentivaram com que a implantação do programa aconteça conforme desejado.

A atuação multiprofissional no Programa Saúde na Escola é um aspecto importante para a efetividade das suas ações, visto que é proposto pela APS a participação dos diversos profissionais que compõem suas equipes no atendimento às necessidades de saúde da população mediante múltiplos olhares.

Observou-se que a participação dos profissionais médicos têm sido realizada em menor quantidade no Município de Natal, o que foi refletido no quantitativo de participantes do presente estudo. Consequentemente isso pode influenciar nas facilidades e dificuldades encontradas no processo de implantação que são referidas de forma diferente por cada profissional.

Dentre as facilidades identificadas, a articulação intersetorial foi a mais frequentemente referida pelos participantes. Ainda que compreendida como a participação de diferentes setores ${ }^{7}$, as respostas obtidas relacionam-na exclusivamente a participação conjunta entre os setores saúde e educação. No entanto, ao referir-se a intersetorialidade, propõe-se que as atividades vão além da participação destes dois setores e que sejam reforçados os vínculos junto às demais setores do território no qual a escola está inserida.

Embora compreendido deste modo, ressaltase a importância deste fator na implantação do Programa Saúde na Escola, proposto para ser executado com atuação compartilhada entre os profissionais de saúde e educação. Mediante isso é possível à aproximação dos setores com compartilhamento de metas a serem obtidas de formar articulada e integrada ${ }^{8}$.

Soma-se à importância de que as atividades do programa estudado necessitam de participação contínua não somente dos profissionais dos setores supracitados, mas que integrem outros envolvidos na comunidade escolar, a exemplos dos pais e responsáveis pelos educandos. Isto certamente contribuirá com o esclarecimento e definição de metas e estratégias que incentivem o convívio social saudável ${ }^{9}$. 
Identificou-se ainda que a satisfação profissional foi fator facilitador para a execução das atividades de saúde escolar. Ressalta-se que estudos têm relacionado este aspecto à resolutividade das atividades, à atuação em equipe e às condições de trabalho nas quais os profissionais estão submetidos, a exemplo da disponibilidade de recursos para executar as atividades ${ }^{10-12}$. Todos estes aspectos corroboram com os achados encontrados no presente estudo, também referidos como fatores facilitadores, ainda que em frequência menor.

A escassez de recursos materiais e financeiros foi referida por mais da metade dos participantes da pesquisa, mostrando que estas necessidades precisam ser supridas na busca pela operacionalização do programa. A característica de necessidade destes recursos também foi identificada em estudo sobre uma intervenção de saúde escolar realizado nos Estados Unidos da América para que ela continuasse sendo executada ${ }^{13}$.

A alocação de recursos materiais e financeiros pode incentivar a mudança de âmbitos concernentes a saúde dos educandos. Exemplo disto são intervenções executadas no México $^{14}$, e na Austrália ${ }^{15}$, que objetivando contribuir com a saúde dos estudantes na prevenção da obesidade focalizaram-se na disponibilização de recursos.

A desarticulação dos profissionais da saúde e educação pode comprometer as atividades propostas pelo Programa Saúde na Escola, a exemplo de estudo que identificou que elas são realizadas principalmente pelos profissionais de saúde e têm os profissionais do setor educação uma participação periférica podendo restringir a potencialidade do programa que desde seu planejamento requer a contribuição das duas partes ${ }^{7}$.

$\mathrm{O}$ excesso de atividades a serem realizadas no processo de trabalho dos profissionais da ESF pode comprometer as atividades do Programa Saúde na Escola. Esta situação também foi observada em estudos referente às ações de saúde na escola que ressaltaram a presença de dificuldades na execução das atividades sob influência da sobrecarga de atribuições ${ }^{16-18}$.

Ressalta-se que esta dificuldade também foi encontrada em todas as regiões brasileiras em estudo que se deteve a identificar os aspectos que contribuíam para a insatisfação dos profissionais da ESF. Observou-se nesse estudo que situações relacionadas à gestão, disponibilidade de recursos e as relações das equipes com os usuários, a exemplo da postura dos usuários contribuem para a ineficácia dos serviços ${ }^{19}$. Estas características corroboram com as dificuldades apontadas pelos participantes no que refere-se à estrutura inadequada para realização das atividades e o desinteresse dos estudantes em participar das atividades.

Também merece destaque à inexistência de capacitações referidas pelos participantes. O programa preconiza que estas aconteçam de modo permanente e contínuo como forma de auxílio na operacionalização do Programa Saúde na Escola. Necessidade semelhante foi encontrada em estudo e sua importância na capacitação e 
educação permanente para com os profissionais dos setores saúde e da educação ${ }^{17}$.

Apesar da frequência de dificuldades ter sido maior em relação às facilidades, reafirma-se a importância do Programa Saúde na Escola enquanto política pública de promoção da saúde. Seu êxito têm sido identificado desde os anos posteriores à sua concepção, onde já em 2010 contava-se com experiências exitosas de sua realização, inclusive com a participação dos profissionais de saúde e educação articulados ${ }^{20}$.

Como limitações ressalta-se o fato de a pesquisa ter focalizado na participação apenas de profissionais do setor saúde. Embora sejam estes os principais responsáveis pela execução da saúde escolar no Brasil, encoraja-se que outras pesquisas possam abranger a compreensão das dificuldades e facilidades também por parte dos profissionais do setor educação.

\section{CONCLUSÕES}

Emmaior frequência foiidentificado que os fatores que comprometem a implantação do programa dizem respeito à escassez de recursos materiais e financeiros, a desarticulação intersetorial e o excesso de atividades no processo de trabalho destes profissionais. Ainda identificou-se que a falta de incentivos por parte da gestão, a impossibilidade de oferecer continuidade no cuidado às pessoas, entre outros fatores também prejudicam a implantação do Programa Saúde na Escola.
Diante destes achados, espera-se que a implantação do programa no Município de Natal e em outros cenários possa ser aprimorada mediante o fortalecimento dos fatores positivos e a readequação dos empecilhos identificados. Para isso, é imprescindível a participação dos profissionais da ESF em articulação com demais setores da sociedade para aprimoramentos das práticas de saúde escolar.

Conflito de interesses: Os autores declaram que não têm nenhum conflito de interesse.

\section{REFERÊNCIAS}

1. Turunen H, Sormunen M, Jourdan D, Seelen JV, Buijs G. Health Promoting Schools - a complex approach and a major means to health improvement. Health Promot Int. 2017; 32(2):177-84. https://doi.org/10.1093/heapro/dax001

2. Fontenele RM, Sousa AI, Rache AS, Souza MHN, Medeiros DC. Participative construction and validation of the logical model of the School Health Program. Saúde Debate. 2017;41(n.esp):167-79. https://doi.org/10.1590/0103-11042017s13

3. Instituto Brasileiro de Geografia e Estatística. Coordenação de População e Indicadores Sociais. Pesquisa Nacional de Saúde do Escolar: 2015. Rio de Janeiro (RJ): IBGE; 2016 [cited 2017 Dez 4]. Available from: http://biblioteca.ibge.gov.br/visualizacao/livros/liv97870. pdf

4. Teixeira MB, Casanova A, Oliveira CCM, Ensgtrom EM, Bodstein RCA. Avaliação das práticas de promoção da saúde: um olhar das equipes participantes do Programa Nacional de Melhoria do Acesso e da Qualidade da Atenção Básica. Saúde Debate. 2014; 38(n.esp):52-68. http://dx.doi.org/10.5935/0103-1104.2014S005

5. Vieira-da-Silva LM. Avaliação de políticas e programas de saúde. Rio de Janeiro: Editora Fiocruz; 2014. https://doi.org/10.7476/9788575415467

6. Van EV, Altman DG, Egger M, Pocock SJ, Gotzsche PC, Vandenbroucke JP. The Strengthening the Reporting of Observational Studies in Epidemiology (STROBE) statement: guidelines for reporting observational studies. Lancet. 2007; 370(9596): 1453-7. https://doi.org/10.1016/S0140-6736(07)61602-X 
7. Sousa MC, Esperidião MA, Medina MG. Intersectorality in the 'Health in Schools' Program: an evaluation of the political-management process and working practices. Ciênc Saúde Coletiva. 2017; 22(6): 1781-90. http://dx.doi.org/10.1590/1413-81232017226.24262016

8. Farias ICV, Sá RMPF, Figueiredo N, Menezes Filho A. Análise da Intersetorialidade no Programa Saúde na Escola. Rev Bras Educ Méd. 2016; 40(2): 261-7. https://dx.doi.org/10.1590/1981-52712015v40n2e02642014

9. Paixão GP, Santos NJ, Matos L, Santos CK, Nascimento DE, Bittencourt I, et al. Violência escolar: percepções de adolescentes. Rev Cuid. 2014; 5(2): 717-22. http://dx.doi.org/10.15649/cuidarte.v5i2.83

10. Lima L, Pires DEP, Forte ECN, Medeiros F. Job satisfaction and dissatisfaction of primary health care professionals. Esc Anna Nery. 2014; 18(1): 17-24. http://dx.doi.org/10.5935/1414-8145.20140003

11. Speroni K, Fruet IM, Dalmolin G, Lima SB. Percepções dos agentes comunitários de saúde: contribuições para a gestão em saúde. Rev Cuid. 2016; 7(2): 1325-37. http://dx.doi.org/10.15649/cuidarte.v7i2.338

12. Tambasco LP, Silva HS, Pinheiro KMK, Gutierrez BAO. Satisfaction in the work of the multidisciplinary team which operates in Primary Health Care. Saúde Debate. 2017; 41(n.spe2): 140-51.

http://dx.doi.org/10.1590/0103-11042017s212.

13. Snelling A, Belson SI, Watts E, Malloy E, Van Dyke $\mathbf{H}$, George S, et al. Measuring the Implementation of a School Wellness Policy. J Sch Health. 2017; 87: 760-8. http://dx.doi.org/10.1111/josh.12548

14. Safdie M, Cargo M, Richard L, Lévesque L. An ecological and theoretical deconstruction of a school-based obesity prevention program in Mexico. Int J Behav Nutr Phys Act. 2014; 11:103. https://doi.org/10.1186/s12966-014-0103-2

15. Lubans DR, Smith JJ, Plotnikoff RC, Dally KA, Okely AD, Salmon J, et al. Assessing the sustained impact of a school-based obesity prevention program for adolescent boys: the ATLAS cluster randomized controlled trial. Int $J$ Behav Nutr Phys Act. 2016; 13:92. https://doi.org/10.1186/s12966-016-0420-8

16. Penso MA, Brasil KCTR, Arrais AR, Lordello SR. A relação entre saúde e escola: percepções dos profissionais que trabalham com adolescentes na atenção primária à saúde no Distrito Federal. Saude Soc. 2013; 22(2): 54253. http://dx.doi.org/10.1590/S0104-12902013000200023

17. Machado MFAS, Gubert FA, Meyer APGFV, Sampaio YPCC, Dias MSA, Almeida AMB, et al. The health school programme: a health promotion strategy in primary care in Brazil. Rev Bras Crescimento Desenvolv Hum. 2015; 25(3): 307-12.

https://dx.doi.org/10.7322/jhgd.96709

18. Sánchez RT, Molina EM, Gómez-Ortega OR. Intervenciones de enfermería para disminuir la sobrecarga en cuidadores: un estudio piloto. Rev Cuid. 2016; 7(1): 1171-84. http://dx.doi.org/10.15649/cuidarte.v7i1.251
19. Soratto J, Pires DEP, Trindade LL, Oliveira JSA, Forte ECN, Melo TP. Job dissatisfaction among health professionals working in the family health strategy. Texto \& Contexto Enferm. 2017; 26(3): e2500016. http://dx.doi.org/10.1590/0104-07072017002500016

20. Santiago LM, Rodrigues MTP, Oliveira Junior AD, Moreira TMM. Implantação do Programa Saúde na escola em Fortaleza-CE: atuação de equipe da Estratégia Saúde da Família. Rev Bras Enferm. 2012; 65(6): 1026-9. http://dx.doi.org/10.1590/S0034-71672012000600020 